\title{
Stationsapotheker sollen Patientenschutz verbessern
}

\section{Deutsche Krankenhausapotheker fordern verpflichtende Einführung}

") Nirgendwo in Europa gibt es so wenige Apotheker in Krankenhäusern wie in Deutschland. Darauf weist der Bundesverband Deutscher Krankenhausapotheker e. V. (ADKA) hin und fordert vom Gesetzgeber die verbindliche Einführung von Stationsapothekern im Krankenhaus.

Zum Vergleich: sind beispielsweise in Großbritannien im Durchschnitt 4,4 Apotheker pro 100 Betten im Krankenhaus beschäftigt, so sind es in Deutschland weniger als 0,4. Dies gehe zu Lasten der Patientensicherheit, warnt der ADKA.
Der „Hochrisikoprozess der Arzneimitteltherapie“ im Krankenhaus brauche als Fachmann den Krankenhausapotheker auf Station. Diese Forderung sei als aktiver Patientenschutz zu verstehen und findet sich auch in den European Statements of Hospital Pharmacy, die einen breiten Konsens von Patienten über Pflegende, Ärzte und Apotheker darstellten.

Der ADKA unterstützt daher die aktuelle niedersächsische Gesetzesinitiative. Der geplante Gesetzesentwurf sieht vor, dass es künftig pro 300 Betten mindestens einen Stationsapothe- ker im Krankenhaus gibt. Er soll die Weiterbildung zum „Fachapotheker für Klinische Pharmazie“ absolviert haben und in der Arzneimitteltherapie bei Aufnahme und Entlassung beraten, ebenso bei der Anwendung und dem Verbrauch von Arzneimitteln und apothekenpflichtigen Medizinprodukten sowie bei der Einhaltung der arzneimittelrechtlichen Bestimmungen.

www.adka.de

\section{GKV und Gesundheitsfonds „Sparkasse der Nation“? \\ DKG fordert Aufnahme der Leistungserbringer in den Schätzerkreis}

" Die Finanzergebnisse des ersten Halbjahres 2017 der Gesetzlichen Krankenversicherung und des Gesundheitsfonds weisen eine "Reserve“ von 17,5 Milliarden Euro aus. Die Deutsche Krankenhausgesellschaft (DKG) spricht von der „Sparkasse der Nation“ und merkt kritisch an, dass Schätzung und Realität in der GKV weit auseinander lägen. Die vorhergesagten Kosten- explosionen im Gesundheitswesen würden offensichtlich nicht eintreten. Nach Einschätzung der DKG zeigen zudem die Zahlen im stationären Bereich, dass bei den Krankenhäusern noch nicht alle Verbesserungen, die im Krankenhausstrukturgesetz vorgesehen waren, wirklich ankommen sind. Der hohe Einnahmeanstieg von 4,3\% mache deutlich, dass eine Reform der
Personalfinanzierungsregelungen für die Krankenhäuser dringend notwendig sei. Daher fordert die DKG nachdrücklich, die Leistungserbringer in den Schätzerkreis aufzunehmen.

\section{Globale Gesundheitsfragen im Fokus: World Health Summit 2017}

\subsection{Teilnehmer aus 100 Ländern in Berlin erwartet}

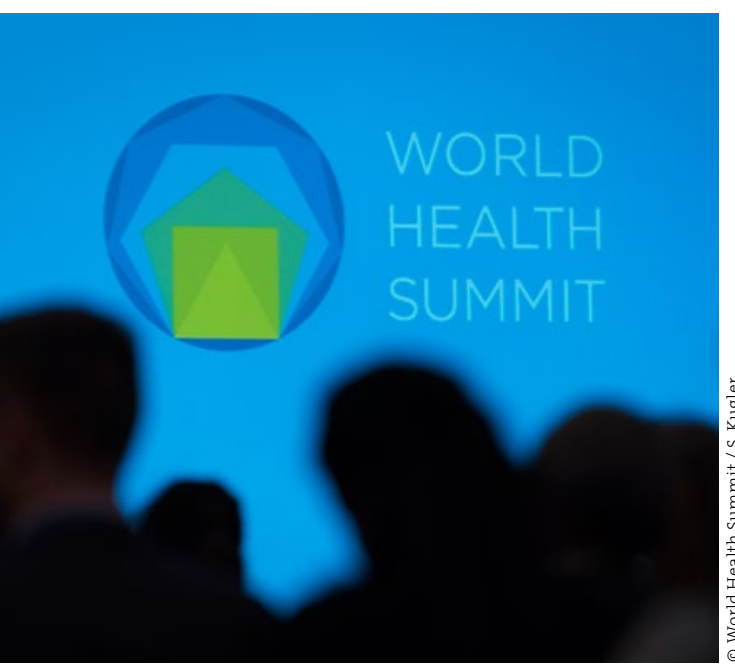

») Vom 15.-17. Oktober 2017 findet der World Health Summit in Berlin statt. Der „Gesundheitsgipfel“ gilt als das wichtigste strategische Forum für weltweite Gesundheitsfragen. Entsprechend international und hochkarätig ist die Konferenz besetzt: Zu den Sprechern zählen acht Minister, zwei Nobelpreisträger sowie CEOs von Unternehmen wie Bayer, Roche und SAP sowie von NGOs, darunter die WHO, das Internationale Rote Kreuz und Ärzte ohne Grenzen .

Die Veranstaltung steht erneut unter der Schirmherrschaft von Bundeskanzlerin Angela Merkel und dem Präsidenten der Europäischen Kom- mission Jean-Claude Juncker. Zentrale Themen 2017 sind die Gesundheitspolitik der G7/G20, die Entwicklung neuer Impfstoffe, die Digitalisierung und Big Data, Gesundheitssicherheit, urbane Gesundheit sowie Afrika und die Nachhaltigkeitsziele der Vereinten Nationen.

Aktuelle Informationen zur Veranstaltung und zum Programm unter

www.worldhealthsummit.org 